\title{
Oxygen and the liberation of placental factors responsible for vascular compromise
}

\author{
Nicola J Robinson, Mark Wareing, Nicola K Hudson, Richard T Blankley, Philip N Baker, John D Aplin \\ and lan P Crocker
}

Maternal endothelial activation in pre-eclampsia is attributed to the release of unknown factors from a hypoperfused placenta. To further characterize these factors, we have used a serum-free placental villous explant culture model and investigated the effect of the liberated soluble factors produced on human endothelial cell cultures. Term placental villous explants from uncomplicated pregnancies were cultured for 4 days in 20,6 or $1 \% \mathrm{O}_{2}$ to mimic placental hyperoxia, normoxia and hypoxia. Medium collected from viable explants was applied to cultured human uterine microvascular endothelial cells. Medium conditioned by hypoxic explants caused a significant decrease in endothelial cell ATP levels and mitochondrial dehydrogenase activity, suggestive of a reduced metabolic rate. An additional reduction in mitochondrial membrane potential and increased endothelial cell death occurred as the oxygen concentration to which explants had been exposed decreased. Effects of the hypoxic explant medium were also seen ex vivo in a wire myography model of myometrial artery function, with increased vasoconstriction and attenuated vasodilation following exposure to hypoxic explant medium. These results suggest that hypoxia $\left(1 \% \mathrm{O}_{2}\right)$ may stimulate the release of soluble factors from the placenta, which have an adverse effect on endothelial cell metabolism and mitochondrial integrity in vitro. These potentially pathogenic factors are now being characterized.

Laboratory Investigation (2008) 88, 293-305; doi:10.1038/labinvest.3700746; published online 28 January 2008

KEYWORDS: endothelium; explant; oxygen; placenta; pre-eclampsia; vascular

There is accumulating evidence for a pathogenic model of pre-eclampsia in which deficient trophoblast invasion of maternal spiral arteries leads to a poorly perfused fetoplacental unit. The hypoperfused placenta is thought to release into maternal circulation factors that cause widespread activation of the vascular endothelium and the clinical syndrome. In vitro studies support the existence of a circulating bioactive factor: for example, exposure of either cultured cells or resistance arteries to plasma from women with pre-eclampsia leads to altered endothelial function. ${ }^{1-5}$

A major risk factor for pre-eclampsia is reduced uteroplacental blood flow in mid-pregnancy, ${ }^{6}$ suggesting that hypoxia could play an important role in the etiology of the disease. Several in vitro studies provide evidence that placental hypoxia is related to pathophysiology. For example, increased trophoblast shedding into the maternal circulation, which occurs in women with pre-eclampsia, is seen when villous tissues are cultured at $2 \% \mathrm{O}_{2} \cdot{ }^{7}$ Changes to the normal trophoblast life cycle occur under hypoxic conditions, evidenced both by increased rates of cytotrophoblast proliferation $^{7}$ and reduced syncytial fusion in vitro. ${ }^{7,8}$ Hypoxia $\left(2 \% \mathrm{O}_{2}\right)$ also inhibits activin A production by trophoblast ${ }^{9}$ and downregulates syncytin expression, ${ }^{10}$ both of which are necessary for fusion. Alterations in the production of vasculogenic mediators such as vascular endothelial growth factor (VEGF), placental growth factor and soluble fms-like tyrosine kinase-1 (sFlt-1), from trophoblast cells occur under reduced oxygen, ${ }^{11,12}$ similar to the alterations in production seen in pre-eclampsia, and these have also been proposed to contribute to disease pathogenesis. ${ }^{13,14}$

A method for identifying pathogenic components in pre-eclampsia would aid our understanding of the disease and could also facilitate the identification of at-risk women. Preliminary characterization studies have demonstrated that the vasoactive factor in plasma is likely to be a protein or associated with a protein. ${ }^{15}$ Plasma complexity, and the fact that a few abundant proteins contribute to over $85 \%$ of its proteome, ${ }^{16}$ makes the direct identification of

Maternal and Fetal Health Research Group, School of Clinical and Laboratory Sciences, The University of Manchester, St Mary's Hospital, Manchester, UK Correspondence: Dr NJ Robinson, PhD, Maternal and Fetal Health Research Group, The University of Manchester, St Mary's Hospital, Manchester M13 0JH, UK. E-mail: nicola.j.robinson@manchester.ac.uk 
low-abundance proteins in maternal blood highly problematic. To test the hypothesis that oxygen regulates the production of soluble factors from the placenta, which influence vascular endothelial cell function, conditioned medium from explants exposed to different oxygen tensions was applied to endothelial cells in culture and bioassays measuring different aspects of endothelial cell function were conducted. The methodology described can form the basis of future protocols to identify pathogenic components of placental origin.

\section{MATERIALS AND METHODS General}

This study was performed with approval from the Ethics Committee of Central Manchester Healthcare Trust for implementation in St Mary's Hospital. Informed written consent was obtained for all tissues used in the study, in compliance with the Helsinki Declaration. Term placentae (37-42 weeks gestation) were obtained at delivery (vaginal or post-elective Caesarean section) from women with otherwise uncomplicated pregnancies. Myometrial biopsies were obtained at delivery by Caesarean section from the upper lip of the lower segment incision, from women with uncomplicated pregnancies. Unless otherwise stated, general chemicals were obtained from Sigma-Aldrich (Poole, UK).

\section{Villous Explant Culture}

Three areas of each placenta were sampled randomly from midway between the chorionic and basal plates, and were dissected within 30 min of delivery. The villous tissue was cut into pieces of similar weight (approximately $5 \mathrm{mg}$ ) and three pieces were cultured on a single individual Netwell support (15-mm diameter, 74- $\mu \mathrm{m}$ mesh; Corning, NY, USA). Cultures were maintained at $37^{\circ} \mathrm{C}$ in a humidified gas mixture of 1,6 or $20 \% \mathrm{O}_{2}$ to mimic physiological and nonphysiological intervillous oxygen tensions, with $5 \% \mathrm{CO}_{2}$ and $\mathrm{N}_{2}$ in $1.5 \mathrm{ml}$ of serum-free microvascular endothelial cell culture medium (TCS Cellworks). Preliminary studies demonstrated that explants were maintained in this serumfree medium for 5 days of culture without loss of viability, as measured by human chorionic gonadotropin secretion and lactate dehydrogenase (LDH) release. ${ }^{17}$ Medium for the explants was changed and replaced with pre-oxygen-conditioned medium every $24 \mathrm{~h}$ for 5 days. The medium was collected from day 4 of culture $(\sim 96 \mathrm{~h})$, when hormone production was rising exponentially. ${ }^{18}$ Collected samples were centrifuged at $3000 \mathrm{~g}$ to remove cellular debris and the supernatants were aliquoted and stored at $-80^{\circ} \mathrm{C}$ for later investigation in the endothelial bioassays.

\section{Cell Culture}

Uterine microvascular endothelial cells (UtMVEC) were obtained from Cambrex (product code CC-2564; Verviers, Belgium) (passage three, and used up to passage ten). These consist of endothelial cells from small uterine vessels, including both spiral arteries and vessels preceding the spiral arteries. The culture medium was the same as that for placental explant cultures (microvascular endothelial cell medium), supplemented with fibroblast growth factor $(20 \mathrm{ng} / \mathrm{ml})$, epidermal growth factor $(10 \mathrm{ng} / \mathrm{ml})$, penicillin $(100 \mathrm{U} / \mathrm{ml})$, streptomycin $(100 \mu \mathrm{g} / \mathrm{ml})$ and $5 \%$ fetal bovine serum (Gibco, Paisley, UK). The cultures were grown as monolayers in a humidified atmosphere of $5 \% \mathrm{CO}_{2}$ at $37^{\circ} \mathrm{C}$. Unless otherwise stated, for all endothelial bioassays, cells were plated into 96-well culture plates at a density of 5000 cells/well in standard medium and left for $24 \mathrm{~h}$ to attach. Medium was removed and the wells washed in phosphate-buffered saline $(\mathrm{PBS})(\times 2)$ before applying 50\% explant-conditioned medium from day 4 of culture (at 20,6 or $1 \% \mathrm{O}_{2}$ ) and $50 \%$ standard serum-free medium.

\section{MTT Test}

The 3-(4,5-dimethylthiazol-2-yl)-2,5-diphenyl tetrazolium bromide (MTT) test estimates mitochondrial oxidative processes in living cells. ${ }^{19}$ Mitochondrial dehydrogenase cleaves MTT to generate a quantitative colorimetric assay of mitochondrial redox potential. A variety of dehydrogenase enzymes in active mitochondria convert the water-soluble tetrazolium dye MTT to its reduced form, resulting in the formation of blue fomazan dye. Cells were cultured in clear 96-well plates (Corning Costar, High Wycombe, UK). An MTT stock solution of $5 \mathrm{mg} / \mathrm{ml}$ in PBS was prepared and added to each well at a quantity equal to one tenth of the total volume. Further incubation was conducted for $3 \mathrm{~h}$ at $37^{\circ} \mathrm{C}$ in an atmosphere of $5 \% \mathrm{CO}_{2}$. At the end of this period, the solution was carefully aspirated and the cells washed with PBS. A $100-\mu$ l volume of dimethylsulfoxide was subsequently added to dissolve the blue-colored formazan particles. The plate was read in a microplate reader at an absorbance of $550 \mathrm{~nm}$.

\section{Measurement of Cellular ATP}

Direct measurements of ATP levels were obtained using the bioluminescent ViaLight kit (Cambrex) based on a luciferase/ luciferin reaction assay. For these experiments, endothelial cells were cultured in clear-bottomed, white-welled, 96-well culture plates (Corning Costar) designed for optimal luminescence measurements, and the bases were covered before taking readings. Following removal of culture medium, nucleotides were released from the cultured endothelial cells by the addition of $50 \mu \mathrm{l}$ of cell lysis reagent. After at least $10 \mathrm{~min}$ at room temperature, $100 \mu \mathrm{l}$ of ATP-monitoring reagent containing luciferin-luciferase was added to each well and the plate was loaded into a Gemini XS fluorescent plate reader (Molecular Probes, Paisley, UK) and luminescence was detected. After 2 min the ATP levels were measured and the results were expressed as relative light units. 


\section{Determination of Mitochondrial Transmembrane Potential}

Mitochondrial transmembrane potential $\left(\Delta \Psi_{\mathrm{m}}\right)$ was determined using the dual-emission mitochondrial dye JC-1, (5,5',6,6' ${ }^{\prime}$-tetrachloro- $1,1^{\prime}, 3,3^{\prime}$-tetraethylbenzimidazolocarbocyanine iodide). JC-1 is a lipophilic, cationic molecule capable of crossing the plasma membrane and specifically binding to the mitochondrial membrane. In the cytosol and the mitochondria, at low $\Delta \Psi_{\mathrm{m}}$ the monomeric form of JC-1 fluoresces green (emission $\sim 525 \mathrm{~nm}$ ), whereas within the mitochondrial matrix at high $\Delta \Psi_{\mathrm{m}}$, JC-1 is able to form aggregates, which fluoresce red (emission $\sim 590 \mathrm{~nm}$ ). Excitation and emission spectra of the dual-emission probe JC-1 were determined using wavelength scans on the Gemini XS fluorescent plate reader. The excitation peak was at $490 \mathrm{~nm}$, with the emission maximum at $530 \mathrm{~nm}$ for the monomer, and the excitation peak and the emission maximum for the aggregate were 530 and $600 \mathrm{~nm}$, respectively. On the basis of these findings, the excitation filter $490 \mathrm{~nm}$ and the emission filter $530 \mathrm{~nm}$ were selected for the green signal, and excitation filter $530 \mathrm{~nm}$ and emission filter $600 \mathrm{~nm}$ were selected for the red signal for further measurements. Cultured cells were grown in black-welled, 96-well plates for fluorescent readings (Corning Costar), and the plate bases were covered before reading. Cells were incubated with JC- 1 at a final concentration of $1 \mu \mathrm{M}$ at $37^{\circ} \mathrm{C}$ for the last $30 \mathrm{~min}$ of the experiment. Red and green fluorescence were quantified using the fluorescent plate reader. Mitochondrial depolarization was induced in the cells by applying different concentrations of the mitochondrial inhibitor antimycin A (5-20 $\mu \mathrm{M})$ for $1 \mathrm{~h}$. Antimycin A inhibits the flow of electrons from cytochrome $b$ to cytochrome $c 1$ in the energy-coupling site of the respiratory system.

\section{Measurement of Endothelial-Cell Apoptosis/Necrosis}

Apoptotic cells were identified by the terminal dUTP nick-end labeling (TUNEL) method, using a commercial kit that labels DNA strand breaks using fluorescein (Roche Diagnostics). UtMVEC were grown on glass coverslips in 24 -well plates. Following incubation with $50 \%$ explant-conditioned medium for $6 \mathrm{~h}$, cells were fixed with $4 \%(\mathrm{v} / \mathrm{v})$ paraformaldehyde in PBS for $20 \mathrm{~min}$ at room temperature. Following three washes in PBS, cells were incubated with permeabilization solution $(0.1 \%$ Triton $(\mathrm{v} / \mathrm{v})$ in $0.1 \%$ sodium citrate $(\mathrm{w} / \mathrm{v})$ in $\left.\mathrm{H}_{2} \mathrm{O}\right)$ for $8 \mathrm{~min}$ and again washed in PBS. Slides were allowed to air dry before applying $40 \mu \mathrm{l}$ of TUNEL reagent per coverslip, and incubating at $37^{\circ} \mathrm{C}$ for $1 \mathrm{~h}$ in a humidified chamber in the dark. The working TUNEL reagent solution was prepared according to the manufacturer's instructions. For positive control slides, sections were treated with $3000 \mathrm{U} / \mathrm{ml}$ DNase I for $10 \mathrm{~min}$ at $25^{\circ} \mathrm{C}$ before the TUNEL reaction. After incubation with TUNEL reagent, coverslips were washed in PBS and mounted using mounting medium containing propidium iodide (Vectashield, Peterborough, UK). Quantification of the number of TUNEL-positive nuclei per field was blinded from a total of six random fields per coverslip. The total number of nuclei per field was also counted, and TUNEL-positive nuclei were expressed as a percentage of the total number of nuclei per microscopic field. A total of 18 images were taken for each condition, using conditioned medium from three separate explants. LDH release from cells exposed to explant medium was determined using a cytotoxicity detection kit (Roche Diganostics, Lewes, UK) according to the manufacturer's instructions.

\section{Angiogenesis Assay}

When seeded onto an extracellular matrix such as Matrigel (BD Biosciences, Oxford, UK), endothelial cells undergo an angiogenic-like process characterized by tubule formation. The length and number of tubules formed provide a simple measurement of angiogenesis. The angiogenic assay was carried out as described previously. ${ }^{20}$ Briefly, UtMVEC were plated onto Matrigel-coated 24-well plates $\left(\sim 3 \times 10^{4}\right.$ per well) in the presence of $50 \%$ explant-conditioned medium from 20,6 or $1 \%$ oxygen cultures, and $50 \%$ microvascular endothelial cell medium. Plates were incubated at $37^{\circ} \mathrm{C}$ for $6 \mathrm{~h}$ under $20 \%$ oxygen, and cells were then fixed using ice-cold $70 \%$ ethanol. Endothelial tubules were visualized by staining with eosin. For each well, eight fields were systematically selected across the plate in an identical pattern. All samples were run in duplicate and a total of 64 images were taken for each oxygen condition.

\section{Wire Myography}

Myometrial biopsies were obtained at delivery by Caesarian section and placed directly in ice-cold physiological salt solution (PSS, containing the following (in $\mathrm{mM}$ ): $119.8 \mathrm{NaCl}$, $25 \mathrm{NaHCO}_{3}, 4.7 \mathrm{KCl}, 2.4 \mathrm{MgSO}_{4}, 1.6 \mathrm{CaCl}_{2}, 1.2 \mathrm{KH}_{2} \mathrm{PO}_{4}, 6.1$ glucose, 0.034 EDTA; pH 7.4). Myometrial small arteries were identified under a stereomicroscope, dissected and carefully cut into length of approximately $2-\mathrm{mm}$ and mounted onto two $40-\mu \mathrm{m}$ steel wires in a wire myograph (M610; Danish Myo Technology, Aarhus, Denmark) as previously described. ${ }^{21}$ Myometrial artery diameter was $274 \pm 20 \mu \mathrm{m}$ ( $n=33$ arteries from nine patients). Following mounting, vessels were bathed in $6 \mathrm{ml}$ of PSS at $37^{\circ} \mathrm{C}$ and normalized to 0.9 of $\mathrm{L}_{13.3 \mathrm{kPa}}$ using the classical normalization procedures described elsewhere. ${ }^{21,22}$ Vessels were continuously gassed in $5 \% \mathrm{CO}_{2}$ in air $\left(<20 \% \mathrm{O}_{2} ; 156 \mathrm{~mm} \mathrm{Hg}\right)$.

\section{Contraction/Relaxation Responses of Myometrial Small Arteries}

Vessel viability was assessed by constriction with $120 \mathrm{mM}$ potassium solution (KPSS; equimolar replacement of $\mathrm{NaCl}$ with $\mathrm{KCl}$ ). Following constriction, vessels were washed to a stable baseline with $6 \mathrm{ml}$ PSS. Dose-response constriction curves to arginine vasopressin (AVP; $0.1-10 \mathrm{nM}$; five doses, 2-min intervals) were constructed, followed by relaxation to bradykinin (BK; 0.01-1000 nM; six doses, 2-min intervals). 
Upon completion of the dose-response curves, vessels were washed to a stable baseline tension using $6 \mathrm{ml}$ PSS, and then washed with $6 \mathrm{ml}$ of $50 \%$ standard culture medium (microvascular endothelial cell medium)/50\% PSS. Pilot experiments indicated that after incubation of vessels in standard cell culture medium for $6 \mathrm{~h}$, contractility was lost, with no response to increasing doses of AVP being apparent (data not shown), and therefore a 2-h incubation period was selected for further analysis. Vessels were incubated with hypoxic $\left(1 \% \mathrm{O}_{2}\right)$ explant-conditioned medium $(50 \%$ explant-conditioned medium, 50\% PSS), or with control medium (50\% culture medium, 50\% PSS). Following incubations, the vessels were washed in PSS and constriction and relaxation to AVP and BK was repeated as outlined above. Vessel tension, expressed as $\Delta \mathrm{T}$ (active wall tension, $\mathrm{mN} / \mathrm{mm})$, was transformed to active effective pressure $(\mathrm{kPa})$ to standardize data by vessel diameter, and then data were expressed as percentage of $\mathrm{KCl}$ constriction to standardize for variations in vessel contractile ability. Relaxation was expressed as a percentage of the maximum constriction to AVP.

\section{Measurement of Release of Vasoactive Substances, Nitrite, Endothelin-1 and Prostacyclin}

Cells were grown in 24-well culture plates at 50000 cells/well, and treated with $50 \%$ explant medium from normoxic, hyperoxic and hypoxic placental explants as described above. After $24 \mathrm{~h}$ of incubation, supernatants were collected, centrifuged to remove cellular debris and stored at $-80^{\circ} \mathrm{C}$ until assay. Nitrite production was determined by a colorimetric assay, following the spontaneous oxidation of nitrite oxide under physiological conditions. This assay is based on the Griess diazotization reaction. ${ }^{23}$ An aliquot of cell supernatant $(150 \mu \mathrm{l})$ was mixed with $20 \mu \mathrm{l}$ Griess reagent $(1 \%$ sulfanic acid and $0.1 \% \mathrm{~N}$-(1-naphthyl)ethylenediamine dihydrochloride in 5\% phosphoric acid; Molecular Probes) in a clear 96-well plate. After incubation at room temperature for $30 \mathrm{~min}$, absorbance was read at $548 \mathrm{~nm}$. A standard curve was generated using serial dilutions of sodium nitrite. Production of prostacyclin $\left(\mathrm{PGI}_{2}\right)$ by endothelial cells was determined by measuring the level of the stable metabolite 6-keto-prostaglandin $\mathrm{F}_{1 \alpha}\left(6-\right.$ keto-PGF $\left.\mathrm{P}_{1 \alpha}\right)$ by a competitive colorimetric enzyme immunoassay (Assay Designs, Cambridge, UK). This assay is based on the competitive binding of 6-keto-PGF $1 \alpha$ with a fixed amount of alkaline phosphatase-labelled 6-keto$\mathrm{PGF}_{1 \alpha}$ for sites on a sheep polyclonal antibody. A quantitative two-step colorimetric sandwich ELISA (R\&D Systems, Abingdon, UK) was used for the quantitative determination of endothelin-1 (ET-1) in the endothelial cell supernatants.

\section{Removal of Abundant Proteins}

Particulate material was removed from the explant medium using a $0.45 \mu \mathrm{m}$ syringe filter. An IgY immunodepletion column (ProteomeLab ${ }^{\mathrm{TM}}$ Spin Column; Beckman Coulter, UK) was then used to simultaneously remove 12 highly abundant proteins from the explant medium. The spin column removed the following: albumin, $\operatorname{IgG}, \operatorname{IgA}$, haptoglobin, transferrin, $\alpha$-1-antitrypsin, fibrinogen, $\alpha$-2-macroglobulin, IgM, $\alpha$-1-acid glycoprotein, apolipoprotein A-I and A-II. The column was run according to manufacturer's instructions apart from substituting the provided Tris-based dilution buffer by PBS.

\section{Measurement of VEGF and sVEGFR-1}

Human VEGF and human sVEGFR-1 were detected in the collected explant-conditioned medium using Quantikine immunoassay kits (R\&D Systems) according to manufacturer's instructions. The minimum detectable doses of either VEGF or sVEGFR-1 using these immunoassays were typically less than 5 and $3.5 \mathrm{pg} / \mathrm{ml}$, respectively. Samples were run in duplicate.

\section{Statistical Analysis}

Data were analyzed using Graphpad Prism (version 4.0; San Diego, CA, USA). Values from the in vitro endothelial bioassays are expressed as medians with interquartile ranges, and, due to the small sample sizes, the statistical significance of differences among the groups was tested using non-parametric one-way Kruskal-Wallis ANOVA or Friedman test for repeated measures, followed by Dunn's post hoc multiple comparisons. All samples were run in triplicate. Myography data are represented as mean \pm s.e.m.; ' $N$ ' refers to the number of patients; ' $n$ ' refers to the number of vessels. Myography data from dose-response curves were transformed using log or arcsine transformation for agonist-induced constriction or relaxation of myometrial small arteries as appropriate. Following transformation, data were compared using the parametric two-way ANOVA test with Bonferroni multiple comparison post hoc analysis. All data were considered significant at $P<0.05$.

\section{RESULTS \\ Effect of Explant-Conditioned Medium on MTT Reduction}

The effects of explant-conditioned medium on mitochondrial redox potential and cellular viability were examined using the MTT test, which measures mitochondrial succinate dehydrogenase activity. MTT reduction following exposure of UtMVEC to explant-conditioned medium for $6 \mathrm{~h}$ was compared with cells in standard control medium. There was a significant decrease in MTT reduction (Figure 1a-c), suggesting a drop in the number of metabolically active cells. As shown in Figure 1d, there was a progressive reduction in metabolic activity following $6 \mathrm{~h}$ exposure to the explant medium as the oxygen tension of the explants decreased. After $24 \mathrm{~h}$ of exposure to the explant medium, the effect of oxygen was lost and the level of MTT reduction was similar (Figure 1e). 

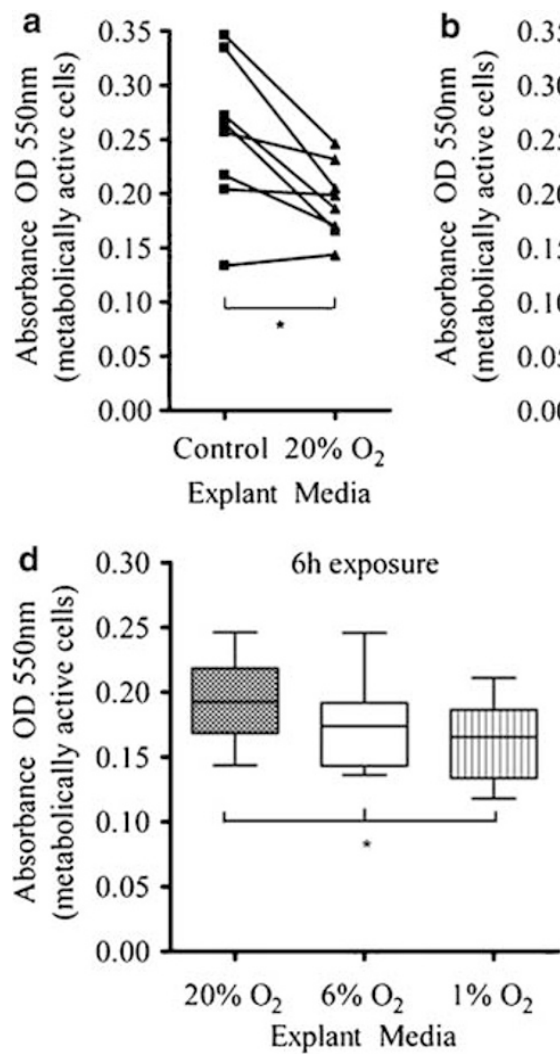
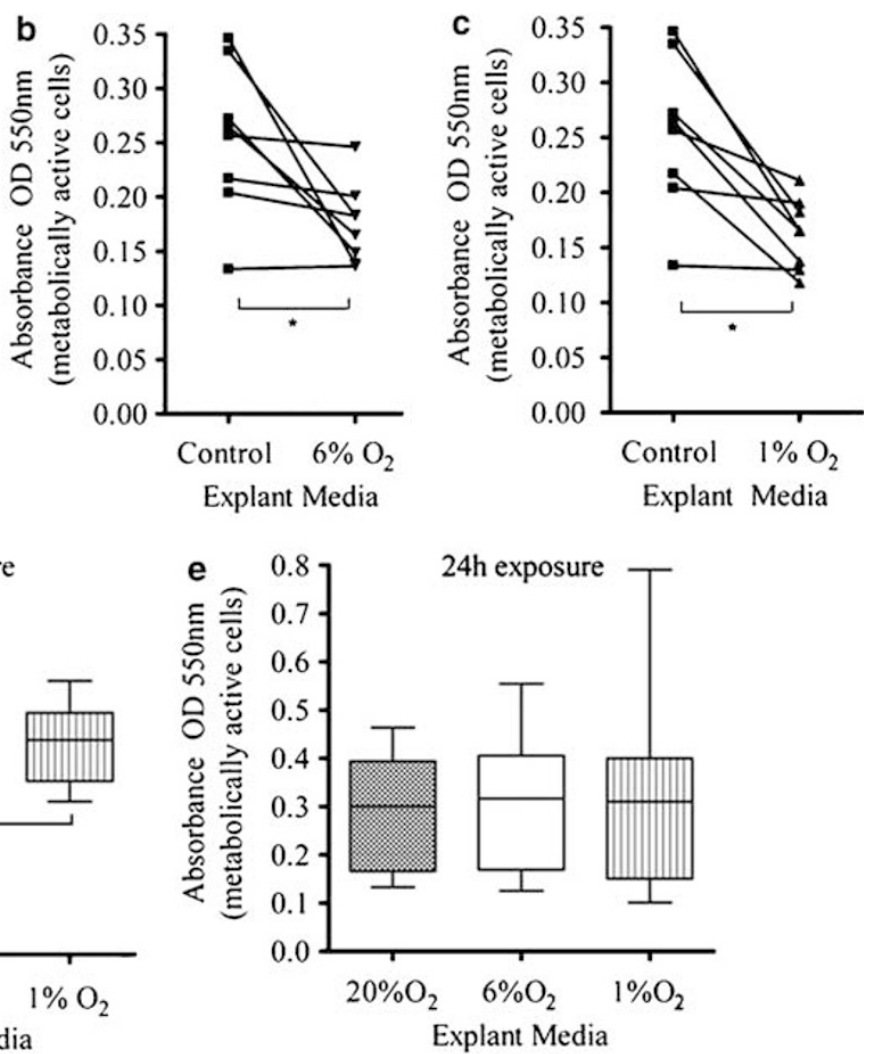

Figure 1 The effect of oxygen-conditioned explant medium on MTT reduction. (a-c) MTT reduction in UtMVEC exposed to 20 (a), 6 (b) or $1 \%$ oxygenconditioned placental explant medium (c) compared with cells exposed to oxygen-conditioned control medium for $6 \mathrm{~h}$ of culture. Treatment with explantmedium from each oxygen tension resulted in a significant decreases in MTT reduction compared with cells incubated with control medium $(P<0.05$ in all cases, Wilcoxon signed-ranks). (d) Reduction of MTT in cells exposed to 20,6 or 1\% oxygen-conditioned medium for $6 \mathrm{~h}$. A significant decrease in MTT reduction occurred as the oxygen tension of the explants decreased $(P<0.05$, Friedman). (e) No significant differences in MTT reduction were observed following exposure of cells to explant-conditioned medium for $24 \mathrm{~h}$. The ends of the boxes define the 25 and 75 th percentiles, the line inside the box defines the median and the whiskers show the maximum and minimum values, $N=8 ;{ }^{\star} P<0.05$.

\section{Effect of Explant-Conditioned Medium on Endothelial-Cell ATP Levels}

To further assess the effect of explant medium on the functional integrity of UtMVEC, cellular ATP levels were determined using a bioluminescence assay. Compared with cells in control medium, a significant decrease in ATP was apparent in the presence of explant-conditioned medium from all oxygen tensions at $6 \mathrm{~h}$ of culture (Figure 2a). The oxygen concentration used had a significant effect on endothelial cell ATP levels. Following $6 \mathrm{~h}$ of exposure, a stepwise reduction in cellular ATP was observed as the oxygen concentration of the explant-conditioned medium decreased (Figure 2b). ATP levels were equivalent in cells exposed to explant medium from different oxygen tensions for the longer incubation period of $24 \mathrm{~h}$ (Figure 2c). After removal of particulate material from the explant-conditioned medium using a $0.45-\mu \mathrm{m}$ spin filter, a trend toward reduced cellular ATP following exposure to hypoxic $\left(\begin{array}{ll}1 \% & \mathrm{O}_{2}\end{array}\right)$ medium, compared with hyperoxic $\left(20 \% \mathrm{O}_{2}\right)$, was apparent (Figure 2d; Mann-Whitney test, $P=0.0556 ; \quad N=5$ ). Similarly, following immunodepletion of abundant proteins from the medium, a significant reduction in ATP levels was maintained following the exposure of UtMVEC to hypoxic compared with hyperoxic explant-conditioned medium (Figure 2d).

\section{Effect of Explant-Conditioned Medium on $\Delta \Psi_{m}$}

To examine the effects of explant-conditioned medium on $\Delta \Psi_{\mathrm{m}}$, endothelial cells were probed with the cationic fluorescent dye JC-1. JC-1 exhibits potential-dependent accumulation in mitochondria, which is indicated by a shift of fluorescence emission from green $(\sim 525 \mathrm{~nm})$ to red $(\sim 590 \mathrm{~nm})$. As demonstrated in Figure 3a, the ratio of red signal to green signal in endothelial cells decreased with increasing concentrations of the mitochondrial inhibitor antimycin. A reduction in the red:green ratio was evident with the lowest antimycin concentration $(5 \mu \mathrm{M}$; Dunn's post-hoc test, $P<0.05)$. After application of explant-conditioned medium to the cells, a reduction in red:green fluorescence ratio was observed as the oxygen concentration of the explant medium decreased (Figure 3b). This reduction in the red:green fluorescence ratio is indicative of mitochondrial depolarization. The decrease in mitochondrial membrane potential in cells exposed to hypoxic $\left(1 \% \mathrm{O}_{2}\right)$ 

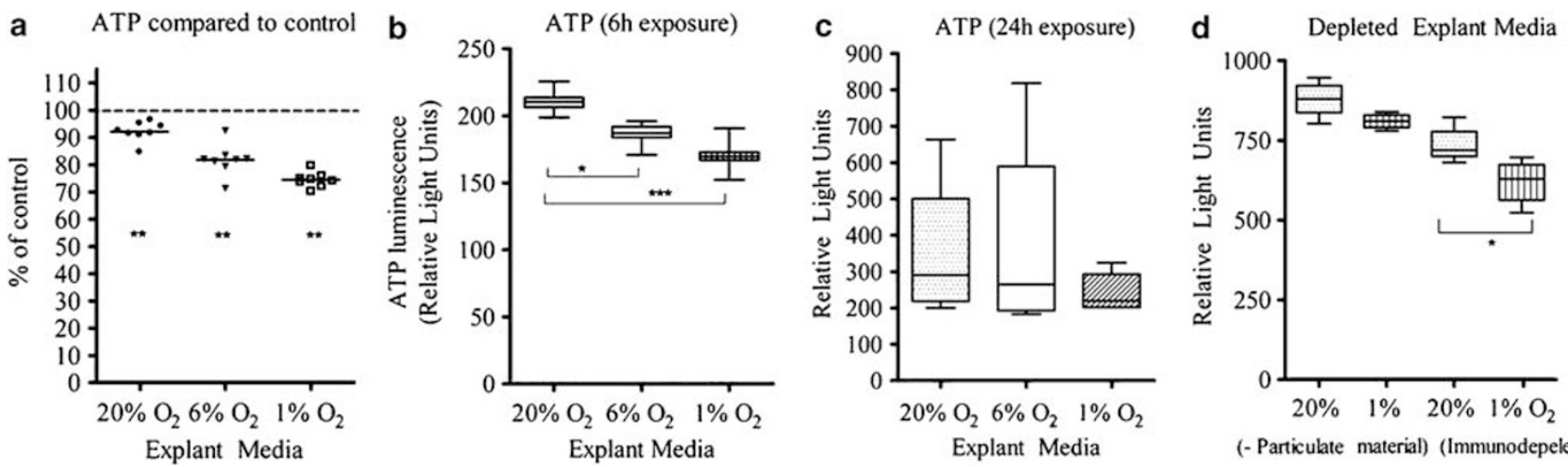

Figure 2 The effect of oxygen-conditioned explant medium on cellular ATP levels. Cellular ATP levels following exposure of UtMVEC to oxygen-conditioned placental explant medium. (a) Exposure of cells to oxygen-conditioned explant medium for $6 \mathrm{~h}$ resulted in significant decreases in cellular ATP levels compared with cells in control medium for each oxygen tension $(P<0.01$ in all cases, Wilcoxon signed-rank test; $N=8)$. Explant medium data are represented as a percentage of cellular ATP levels in control medium (dotted line). (b) The oxygen concentration of the placental explants had a significant effect on cellular ATP levels following $6 \mathrm{~h}$ exposure, with a stepwise reduction in ATP as the oxygen concentration of the explant medium decreased $(P<0.001$ Kruskal-Wallis test followed by Dunn's post hoc analysis; $N=8)$. (c) No significant differences in cellular ATP levels were determined following exposure to explant conditioned medium for $24 \mathrm{~h}(N=8)$. (d) Following immunodepletion of abundant proteins from explant conditioned medium, the reduction in ATP following exposure to hypoxic $\left(1 \% \mathrm{O}_{2}\right)$ explant medium was still apparent $(P<0.05$, Mann-Whitney test; $N=5)$. ${ }^{\star} P<0.05 ;{ }^{*} P<0.01$; $* * * P<0.001$.
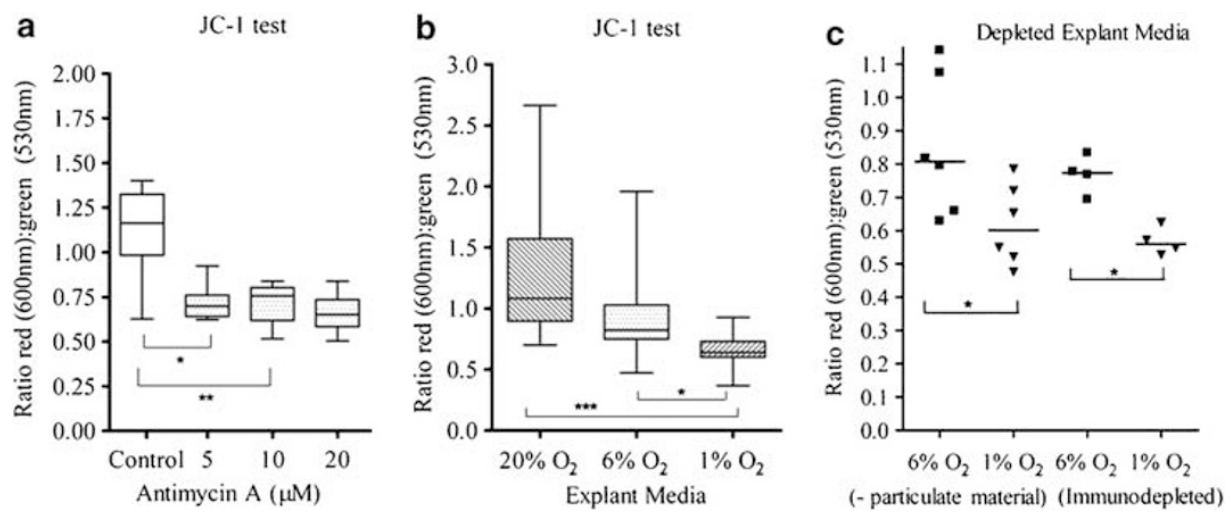

Figure 3 The effect of oxygen-conditioned explant medium on JC-1 fluorescence. (a) A reduction in the JC-1 red:green fluorescence ratio was demonstrated following exposure of cells to $5-20 \mu \mathrm{M}$ antimycin A. Asterisks indicate values significantly different from untreated cells $(P<0.05$ and $P<0.01$, Kruskal-Wallis test and Dunn's post hoc analysis). (b) Application of explant-conditioned medium to the cells resulted in a reduction in red:green fluorescence ratios as the oxygen concentration of the explant medium decreased $(P<0.001$, Kruskal-Wallis test and Dunn's post hoc analysis; $N=12)$. (c) The reduction in the red:green fluorescence ratio following cell exposure to hypoxic $\left(1 \% \mathrm{O}_{2}\right)$ in comparison with normoxic $\left(6 \% \mathrm{O}_{2}\right)$ explant medium was maintained following the removal of particulate material $(P<0.05$, Mann-Whitney test; $N=6)$ and subsequent immunodepletion of abundant proteins $(P<0.05$, Mann-Whitney test; $N=4) .{ }^{*} P<0.05 ; * * P<0.01 ;{ }^{* * * P<0.001 .}$

compared with normoxic $\left(6 \% \mathrm{O}_{2}\right)$ medium was maintained following both the removal of particulate material using a $0.45-\mu \mathrm{m}$ filter (Figure 3c; Mann-Whitney test, $P<0.05$; $N=6)$ and the subsequent immunodepletion of abundant proteins from the medium (Figure 3c; Mann-Whitney test, $P<0.05 ; N=4)$, as evidenced by a decreased red:green fluorescent ratio.

\section{Effect of Explant-Conditioned Medium on Cellular Apoptosis/Necrosis}

Altered mitochondrial membrane potential may be suggestive of the early stages of apoptosis. To determine the effect of explant-conditioned medium on endothelial cell death, TUNEL staining was carried out. Figure $4 \mathrm{a}$ shows representative immunofluorescence images of cells exposed for $6 \mathrm{~h}$ to 20,6 or $1 \% \mathrm{O}_{2}$-conditioned explant medium. TUNEL-positive nuclei (green) were expressed as a proportion of the total number of nuclei (red) per field (Figure 4b). Very few or no TUNEL-positive nuclei were seen after exposure of cells to medium conditioned with either 20 or $6 \%$ oxygen. Compared with either 20 or $6 \% \mathrm{O}_{2}$, medium from placental explants conditioned under hypoxia $\left(1 \% \mathrm{O}_{2}\right)$ significantly increased the proportion of TUNEL-positive nuclei, suggestive of an increase in cell death. LDH release 
a
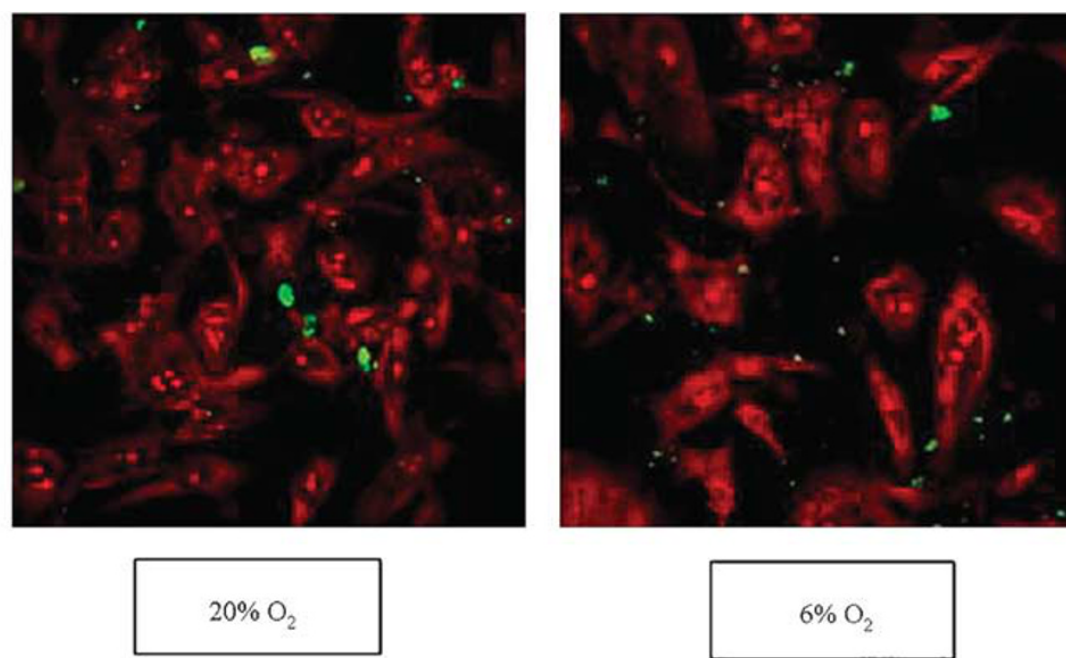

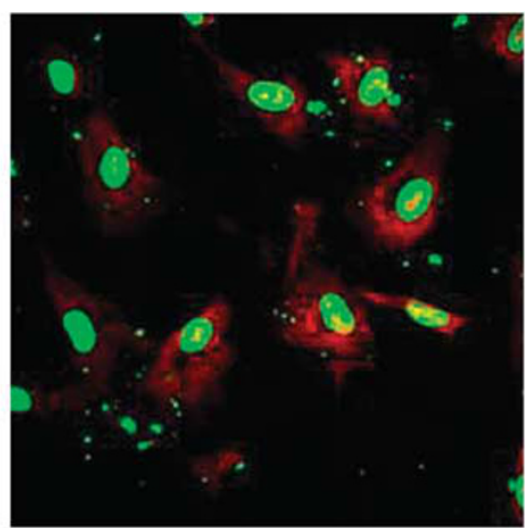

$1 \% \mathrm{O}_{2}$
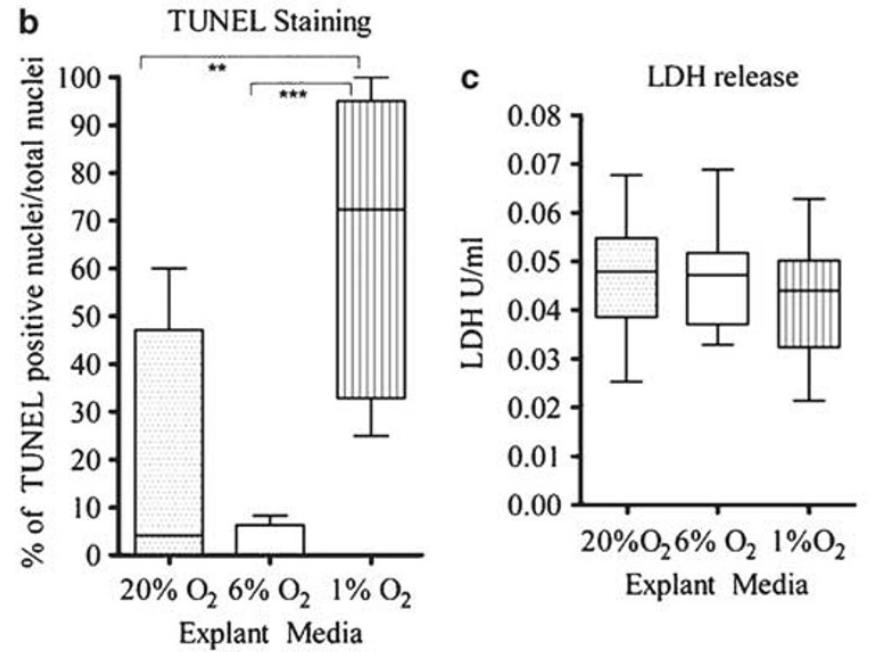

Figure 4 The effect of oxygen-conditioned explant medium on cell death. (a) TUNEL staining of cultured UtMVEC exposed to 20, 6 or 1\% oxygen-conditioned explant medium for $6 \mathrm{~h}$. Nuclei fluoresce red, while TUNEL-positive nuclei fluoresce green. (b) TUNEL-positive nuclei were expressed as a proportion of the total number of nuclei per field. Cells exposed to hypoxic $\left(1 \% \mathrm{O}_{2}\right)$ explant medium displayed in increased TUNEL-positive nuclei $(P<0.001$ Kruskal-Wallis test and Dunn's post hoc analysis) $(N=3, n=18)$. (c) No significant changes in LDH release occurred from cells exposed to explant medium from differing oxygen tensions $(N=9) .{ }^{* *} P<0.01 ;{ }^{* *} P<0.001$.

from the cells was measured as a marker of necrosis following exposure to explant-conditioned medium. There was no change in $\mathrm{LDH}$ release when cells were exposed to explant medium under differing oxygen concentrations (Figure 4c).

\section{Effect of Explant-Conditioned Medium on Angiogenesis}

UtMVEC cultured on Matrigel were exposed to explant medium conditioned with 20,6 or $1 \% \mathrm{O}_{2}$. Figure 5 shows that cells in hypoxic explant medium $\left(1 \% \mathrm{O}_{2}\right)$ had reduced average tubule length compared with cells exposed to 20 or $6 \% \mathrm{O}_{2}$ explant medium. Hypoxic medium also caused a slight increase in tubule number relative to hyperoxic medium.

\section{Effect of Explant-Conditioned Medium on Vasoconstriction of Myometrial Arteries Ex Vivo}

Myometrial small-artery contractility ex vivo, in response to AVP is shown in Figure 6. Following a $2 \mathrm{~h}$ incubation, there was a significant decrease in vessel contractility compared with the constriction produced before the application of culture medium, in vessels exposed either to control (unconditioned) or hypoxic explant medium (Figure 6a and b). The data demonstrate that standard culture medium has a detrimental effect on the vessel response, perhaps due to differences in the ion content of the culture medium compared to PSS, the usual assay buffer for such experiments. ${ }^{5,21,22}$ Microvascular endothelial cell culture medium has the same $\mathrm{Ca}^{2+}$ concentration as PSS $(1.6 \mathrm{mM})$, 

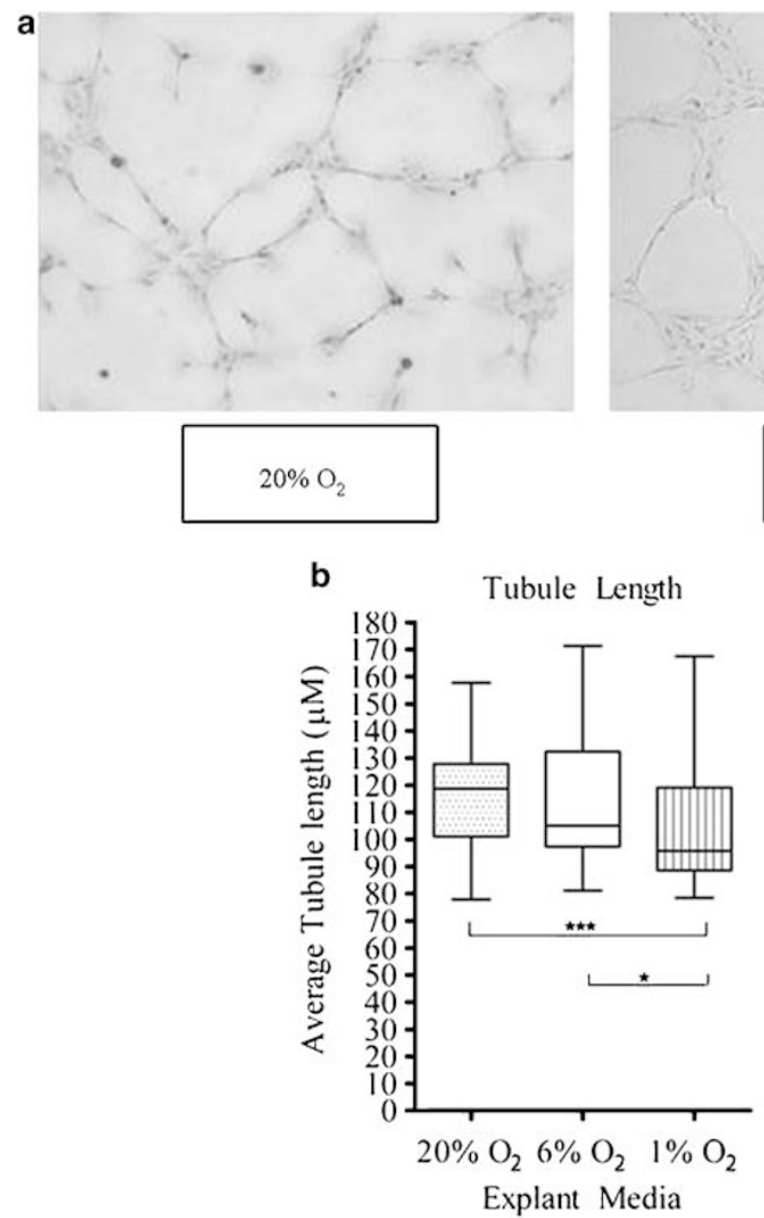
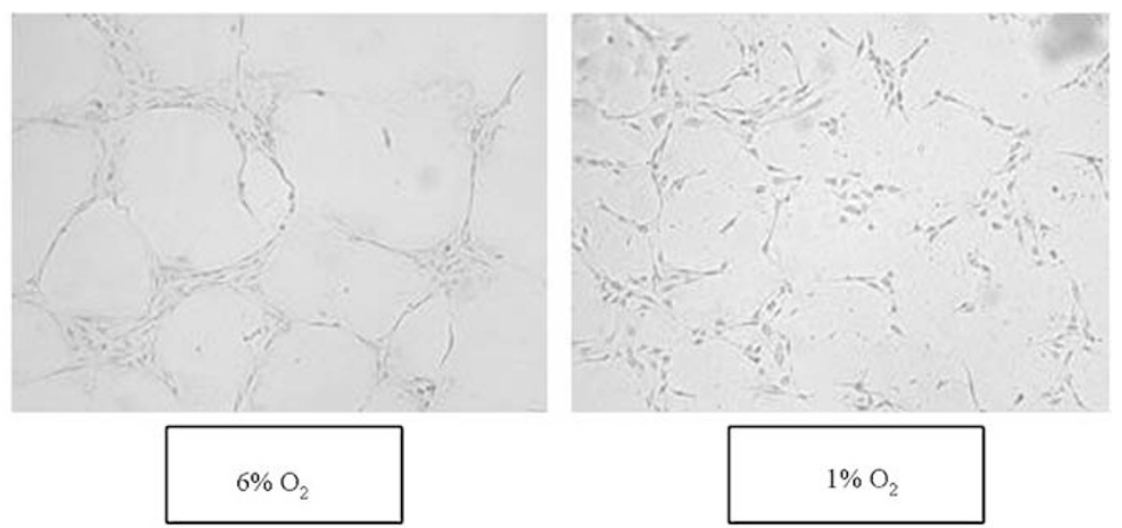

c

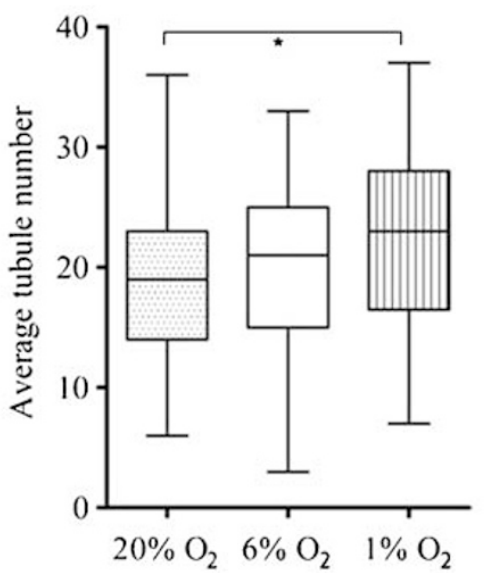

Explant Media

Figure 5 The effect of oxygen-conditioned explant medium on angiogenesis. (a) Light micrographs showing the appearance of tubules formed by UtMVEC seeded on Matrigel with either 20,6 or $1 \%$ oxygen-conditioned explant medium. Tubule length (b) and tubule number (c) were determined by image analysis. Hypoxic explant medium $\left(1 \% \mathrm{O}_{2}\right)$ caused both a significant reduction in average tubule length $(\mathbf{b})(P<0.001$ Kruskal-Wallis test and Dunn's post hoc analysis) and an increase in the average tubule number (c) $\left(P<0.05\right.$ Kruskal-Wallis test and Dunn's post hoc analysis), $N=4, n=64 .{ }^{\star} P<0.05$; $* * * P<0.001$.

but four times higher levels of $\mathrm{Mg}^{2+}(10 \mathrm{mM}$ medium, $2.4 \mathrm{mM}$ PSS). However, the residual response was sufficient to compare the properties of explant medium generated at differing oxygen concentrations. Vasoconstriction was significantly enhanced after exposure to hypoxic explant medium, compared with that seen after exposure to control medium (Figure 6c). This suggests that exposure to hypoxic explant medium modifies vasoconstriction of myometrial small arteries.

\section{The Effect of Explant-Conditioned Medium on Vasodilatation of Myometrial Arteries Ex Vivo}

AVP-constricted myometrial small arteries exhibited dose-dependent relaxation to increasing concentrations of BK (Figure $6 \mathrm{~b}$ ). Following $2 \mathrm{~h}$ of exposure to standard culture medium, enhanced relaxation was observed compared with freshly isolated vessels. This was also evident in vessels exposed to hypoxic explant medium (Figure 6b(i) and (ii)). Exposure of the vessels to hypoxic explant medium resulted in a significant attenuation of endothelium-dependent relaxation, compared with vessels exposed to control medium (Figure 6b(iii)).

\section{The Effects of Explant-Conditioned Medium on Endothelial-Cell $\mathrm{PGI}_{2}$, Nitrite and ET-1 Production}

The release of vasoactive metabolites from cultured endothelial cells was assessed after incubation with explant-conditioned medium. Media under all oxygen concentrations stimulated UtMVEC to produce the vasodilator 6-keto- $\mathrm{PGF}_{1 \alpha}$ over the 24 -h incubation period (Figure 7a). Similarly, all media stimulated an equivalent rate of nitrite release from the endothelial cells (Figure $7 b$ ). The release of the vasoconstrictor ET-1 from cultured endothelial cells over the 24-h incubation period was also unaltered following exposure to hypoxic explant medium (Figure 7c). No statistically significant differences were therefore seen in the release of any vasoactive metabolite tested, following exposure of endothelial cells to explant medium from differing oxygen concentrations. 
a (i)

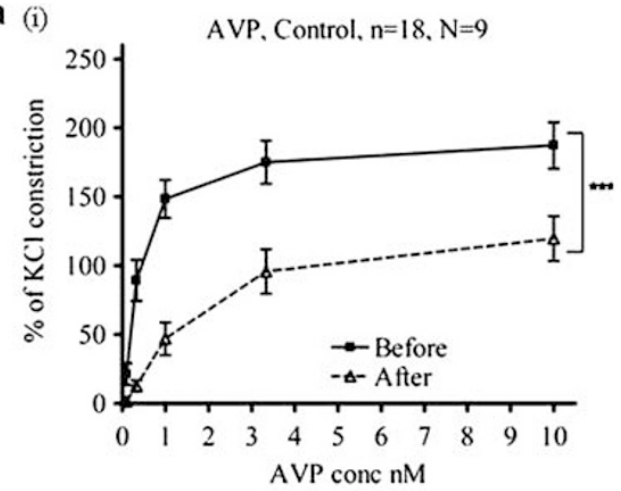

(ii)

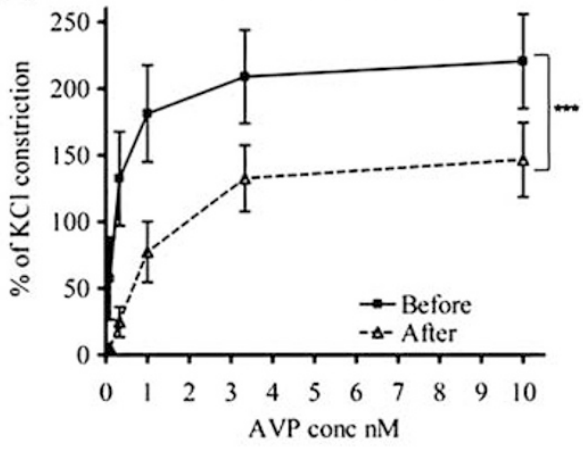

(iii)

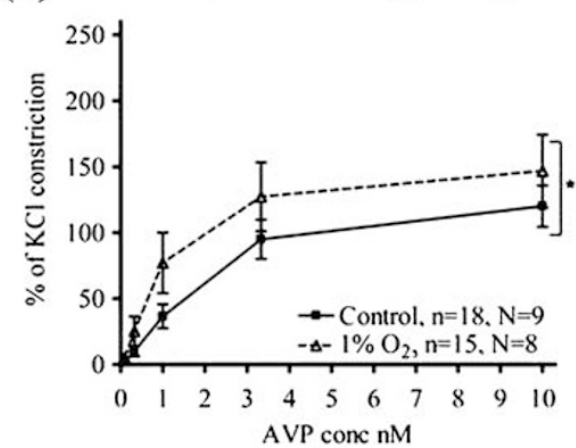

b (i)

BK. Control, $n=18, N=9$

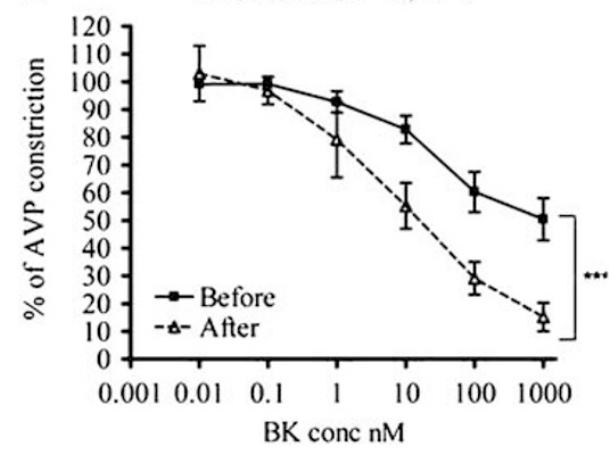

(ii)

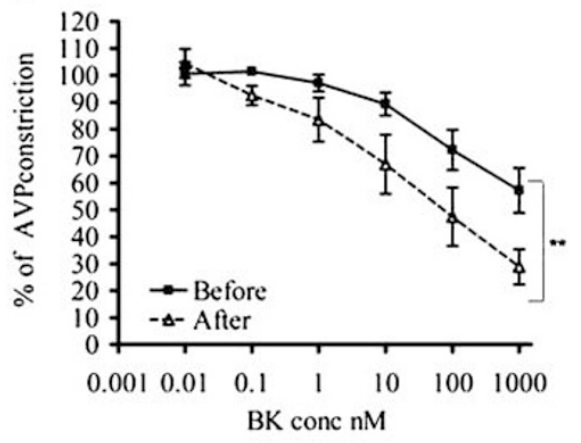

(iii)

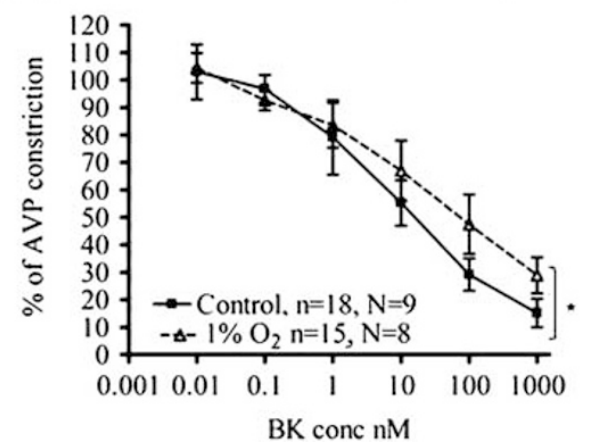

Figure 6 The effect of oxygen-conditioned explant medium on constriction and relaxation of myometrial arteries. Concentration-response curves for AVP-induced constriction (\% of $\mathrm{KCl}$ constriction) (a) and BK-induced relaxation (\% of maximum constriction) (b) of myometrial arteries. A 2-h incubation in either control medium (a, i; $N=9, n=18$ ) or in hypoxic-explant medium (a, ii; $N=8, n=15$ ) resulted in a significant reduction in constriction (open triangle, dotted line) compared with the preincubation curve (closed square, solid line; $P<0.001$, two-way ANOVA in both cases). (a, iii) Exposing the arteries to hypoxic explant medium (open triangle, dotted line) significantly shifted the concentration-response curve for AVP upwards, compared with arteries exposed to control medium (closed square, solid line; $P<0.05$, two-way ANOVA). Exposure of myometrial arteries for $2 \mathrm{~h}$ to either control medium (b, i; $N=9, n=18$ ) or hypoxic-explant medium (b, ii; $N=8, n=15$ ) (both open triangle, dotted line) resulted in enhanced vasodilatation in response to BK, compared with the pre-medium relaxation curve (closed square, solid line; $P<0.001$ and $P<0.01$, respectively, two-way ANOVA). (b, iii) The BK-induced relaxation of myometrial arteries exposed to hypoxic explant medium (open triangle, dotted line) for $2 \mathrm{~h}$ was significantly greater than that of

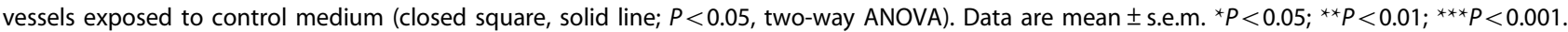

\section{Levels of VEGF and SVEGFR-1 in the Explant-Conditioned Medium}

VEGF and its soluble receptor sVEGFR-1 were measured in the explant-conditioned medium. Detectable levels of sVEGFR-1 were present in media under all oxygen concentrations, with no significant changes in levels as the oxygen tension altered (Figure 8a). Levels of free VEGF were undetectable in the explant-conditioned medium in ELISA. Levels of sVEGFR-1 in the explant medium far exceed the levels reported to interfere with the VEGF immunoassay $(\geq 500 \mathrm{pg} / \mathrm{ml})$, and this may therefore account for the inability to measure free VEGF. 

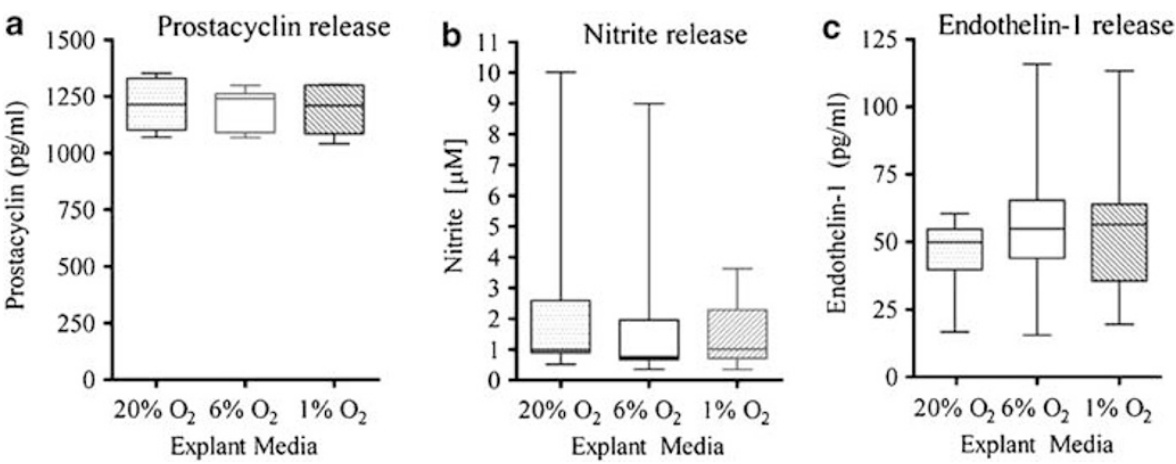

Figure 7 The effect of oxygen-conditioned placental medium on the release of vasoactive metabolites. The release of vasodilators 6-keto-PGF $1 \alpha(\mathbf{a})$ and nitrite (b), and vasoconstrictor ET-1 (c), from cultured UtMVEC following the application of oxygen-conditioned placental explant medium. No significant changes in release were determined as the oxygen concentration decreased; $N=8$.

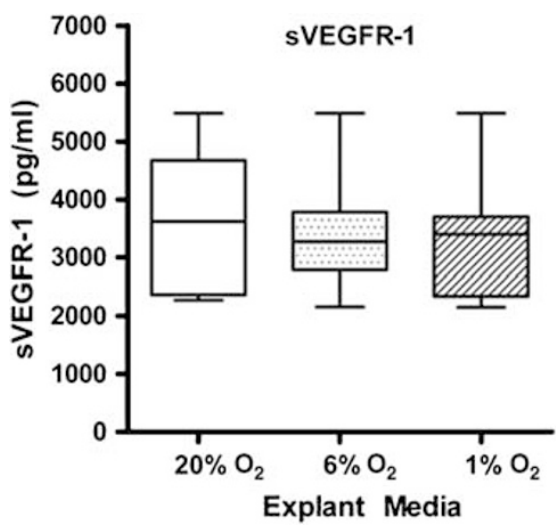

Figure 8 The measurement of liberated VEGF and SVEGFR-1 from the placental explants. Free VEGF and its soluble receptor SVEGFR-1 were measured in the explant-conditioned medium by immunoassay. Free VEGF was undetectable (not shown), but sVEGFR-1 was present in explant medium under all oxygen concentrations, with no significant changes in levels as the oxygen tension altered; $N=8$.

\section{DISCUSSION}

Plasma complexity hinder the characterization of bioactive or pathogenic factors in maternal blood. We have optimized serum-free conditions for the culture of placental villous explants and the subsequent testing of liberated factors on primary endothelial cell function. Explant cultures from human placental tissue derived at term provide a mechanism for examining the effects of key variables such as oxygen concentration on the release of bioactive factors from the placenta. Under normal physiological conditions, intervillous oxygen tensions at term have been estimated at $45-50 \mathrm{~mm} \mathrm{Hg}$, which can be replicated in vitro using 5-6\% ambient oxygen levels. ${ }^{24,25}$ Variations in oxygen concentration in culture were used to replicate the suggested conditions of the placenta in pre-eclampsia, thereby stimulating events which may encourage protein liberation. 6\% oxygen was thought to represent normoxia for the placenta at term, and 20 or $1 \%$ oxygen levels were used for either hyperoxia or hypoxia, either of which may be relevant to the aberrant perfusion of the placenta in pre-eclampsia.
This study has demonstrated that hypoxia $\left(\begin{array}{lll}1 \% & \mathrm{O}_{2}\end{array}\right)$ stimulates the liberation of factors from the placenta, which influence the metabolic status and the mitochondrial integrity of microvascular endothelial cells in vitro. Decreasing oxygen concentrations used during explant culture significantly altered the release of factors that affected viability, metabolism, angiogenic properties and cell death of primary cultured endothelial cells. These findings support a model of pre-eclampsia in which soluble factors released by the placenta cause the generalized endothelial pathology associated with the disease, and demonstrate the suitability of the explant model for studies of placenta-dependent pregnancy pathology.

Following exposure to explant-conditioned medium from each oxygen tension, a decreased rate of MTT reduction was seen when compared with cells in standard culture medium. Compared with medium under other oxygen tensions, the rate of MTT reduction was relatively lower following exposure of endothelial cells to hypoxic placental explant medium, indicating a greater alteration of oxidative metabolism and a decrease in the mitochondrial redox potential. An acute effect of the hypoxic explant medium on endothelial cell metabolic function was evident, with no changes in MTT reduction (or cellular ATP levels) after the longer 24-h exposure time, suggesting some population recovery. A decrease in MTT reduction was previously demonstrated in human umbilical vein endothelial cells exposed to placental extracts from women with preeclampsia compared with normotensive women, ${ }^{26}$ and this effect was reduced by trypsin digestion and heat treatment.

The damage of mitochondrial integrity or the loss of $\Delta \Psi_{\mathrm{m}}$ is an early event in the initiation and activation of apoptotic cascades. ${ }^{27,28}$ During this process, the electrochemical gradient across the mitochondrial membrane collapses. In this study, the fluorescent cationic carbocyanine dye, JC-1, was used to signal the loss of $\Delta \Psi_{\mathrm{m}}$. Hypoxic explant medium caused a significant disruption of $\Delta \Psi_{\mathrm{m}}$, as demonstrated by a decrease in the JC-1 aggregate-to-monomer ratio. Further evidence for induction of apoptosis in the endothelial cells 
following exposure to hypoxic explant medium was obtained using the TUNEL assay.

The electrochemical gradient across the mitochondrial membrane collapses during the early stages of apoptosis. This collapse is thought to occur through pore formation in mitochondria by proapoptotic regulators such as dimerized Bax, or activated Bid, Bak or Bad proteins, ${ }^{29-32}$ causing cytochrome $c$ release into the cytoplasm and ultimately resulting in caspase activation. The balance between the antiapoptotic Bcl-2, which prevents cytochrome $c$ release from mitochondria, and the proapoptotic Bax, is thought to be important in controlling apoptosis. Increased Bax expression and decreased $\mathrm{Bcl}-2$ expression have previously been demonstrated from cultured cytotrophoblasts under hypoxic conditions. ${ }^{33,34}$ In pre-eclampsia, which is associated with altered trophoblast cell turnover and increased apoptosis, altered expression of pro- and antiapoptotic factors have also been reported. ${ }^{35,36}$ Changes in the expression of pro- and anti-inhibitory proteins such as Bax and Bcl-2 could provide a possible mechanism for the alteration in mitochondrial membrane potential and increased cell death following exposure of the endothelial cells to hypoxic-conditioned explant medium seen here.

Mitochondrial membrane potential can be used as an indicator of cellular viability, reflecting the flow of hydrogen ions across the mitochondrial inner membrane during the electron transport chain, and the generation of ATP through oxidative phosphorylation. We have previously shown that plasma from women with pre-eclampsia causes a significant reduction in endothelial cellular ATP levels. ${ }^{37}$ This was accompanied by a simultaneous increase in the activity of the nuclear enzyme poly(ADP-ribose) polymerase (PARP). Application of the synthetic PARP inhibitor PJ34 reversed the effect of the pre-eclamptic plasma on endothelial cellular ATP levels, implying that PARP activation played a significant role in the pathogenesis of endothelial dysfunction in pre-eclampsia. Here, restricted oxygen (1\%) significantly enhanced the capacity of placental-conditioned medium to reduce ATP in endothelial cultures. In vivo, following cytotrophoblast differentiation and fusion to form syncytiotrophoblast, terminal differentiation occurs within the syncytium. This eventually leads to packaging of apoptotic material into syncytial knots and smaller vesicular structures, which are shed into maternal circulation. In normal pregnancy, trophoblast cells and syncytiotrophoblast microparticles are spontaneously shed from the placenta into the maternal circulation as part of the of normal placental physiologic turnover. ${ }^{38-40}$ Syncytiotrophoblast membrane microparticles have previously been prepared in vitro from term placental villous explant cultures. ${ }^{41}$ Furthermore there is evidence that microparticulate material can affect endothelial integrity. ${ }^{42}$ In the present study, following the removal of particulate material, exposure to hypoxic explant medium still resulted in both an increase in cell death, as evidenced by mitochondrial depolarization, and reduced endothelial cellular ATP levels. Thus, it appears that placental factor(s) other than shed microparticles can affect endothelial cell metabolism. Immunodepletion of 12 highly abundant proteins (including albumin, transferrin and fibrinogen) did not reduce the effect of the hypoxia-conditioned explant medium on mitochondrial membrane polarization or cellular ATP. This suggests that the active factor liberated under hypoxia is not associated with these abundant proteins.

Hypoxia is known to have a significant effect on early placental development, with low placental oxygen in early pregnancy promoting branching angiogenesis and hypercapillarization, resulting in a majority of intermediate villi. ${ }^{43}$ This is an effect similar to that occurring in vivo at high altitude and with maternal anemia. ${ }^{44}$ As pregnancy develops, uterine blood flow increases, resulting in a predominance of non-branching angiogenesis. Oxygen regulation of placental vascularization is thought to be mediated by angiogenic factors, including VEGF, PLGF and angiopoietin- $1 .{ }^{45-47}$ Previous experiments have shown that placental explant medium conditioned at $3 \% \mathrm{O}_{2}$ significantly decreased tubule length and increased branching in HUVEC cells, compared with medium under $20 \% \mathrm{O}_{2}$, and this effect could be blocked using neutralizing antibody to VEGF. ${ }^{20}$ Current data using UtMVEC with explant-conditioned medium from differing oxygen tensions support these findings. sVEGFR-1 was present in the explant medium, but its levels were not altered by culture under differing oxygen tensions. Free VEGF was undetectable in the explant medium. The VEGF sandwich ELISA measures the combined free-form levels of the VEGF splice variants VEGF-121, -145, and -165 , and the inability to detect it in the explant medium suggests that VEGF may be sequestered by soluble receptor. The levels of sVEGFR-1 $\left(6 \% \mathrm{O}_{2}, 3.4 \pm 0.36 \mathrm{ng} / \mathrm{ml}\right)$ in placental explant medium were similar to those previously measured in maternal sera from normal pregnancies $(1.8 \pm 0.48 \mathrm{ng} / \mathrm{ml}){ }^{48}$ Our findings are consistent with those of Brownbill et al (2007), ${ }^{49}$ who determined, using a placental perfusion model, that free VEGF was not present in the maternal circulation.

A further question to address was whether soluble factors from placenta alter vessel function. Hypoxic explant medium increased the vasoconstriction response to AVP in comparison with vessels exposed to standard medium. AVP promotes constriction by direct (endothelium-independent) stimulation of $V_{1}$ receptors on vascular smooth muscle. ${ }^{50}$ We have previously demonstrated that plasma from women with pre-eclampsia is capable of reducing endothelium-dependent relaxation of myometrial vessels from normal pregnant women. ${ }^{3}$ In the present study, hypoxic explant medium also decreased vessel relaxation in response to BK. Increased vasoconstriction and reduced vasodilatation of myometrial vessels exposed to hypoxic explant medium failed to correlate with changes in the release of vasoactive metabolites (eg, nitrite, ET-1, 6-keto-PGF ${ }_{1 \alpha}$ ) from cultured endothelial cells. 
A possible explanation for the effect seen on vascular reactivity is that factors present in the hypoxic explant-conditioned medium are capable of altering the sensitivity of the smooth-muscle receptors to the vasoactive agents. This mechanism is the focus of current investigations.

In summary, this study demonstrates that hypoxic conditions stimulate from placental villous tissue the secretion of soluble factors that are capable of affecting endothelial metabolic status and mitochondrial integrity, and also alter vascular function. It is hoped that placental explants can be used to identify bioactive soluble factors that are released into maternal circulation. We aim to combine high-throughput endothelial bioassays with fractionation, depletion and mass spectrometric techniques to identify secreted factors responsible for the metabolic compromise of vascular endothelial cell status in pre-eclampsia.

\section{ACKNOWLEDGEMENT}

We gratefully acknowledge the financial support of Sport Aiding Medical Research for Kids (SPARKS) and Tommy's the Baby Charity. We would also like to thank the doctors, midwives and the patients at St Mary's Hospital for their cooperation during this study. IC is a recipient of an NCC RCD Personal Award from the Department of Health, UK.

1. Baker PN, Davidge ST, Barankiewicz J, et al. Plasma of preeclamptic women stimulates and then inhibits endothelial prostacyclin. Hypertension 1996;27:56-61.

2. Baker PN, Davidge ST, Roberts JM. Plasma from women with preeclampsia increases endothelial cell nitric oxide production. Hypertension 1995;26:244-248.

3. Ashworth JR, Warren $A Y$, Johnson IR, et al. Plasma from pre-eclamptic women and functional change in myometrial resistance arteries. $\mathrm{Br} J$ Obstet Gynaecol 1998;105:459-461.

4. Aalkjaer $\mathrm{C}$, Danielsen $\mathrm{H}$, Johannesen $\mathrm{P}$, et al. Abnormal vascular function and morphology in pre-eclampsia: a study of isolated resistance vessels. Clin Sci (Lond) 1985;69:477-482.

5. Hayman R, Warren A, Brockelsby J, et al. Plasma from women with preeclampsia induces an in vitro alteration in the endothelium-dependent behaviour of myometrial resistance arteries. BJOG 2000;107:108-115.

6. Chappell L, Bewley S. Pre-eclamptic toxaemia: the role of uterine artery Doppler. Br J Obstet Gynaecol 1998;105:379-382.

7. Huppertz B, Kingdom J, Caniggia I, et al. Hypoxia favours necrotic versus apoptotic shedding of placental syncytiotrophoblast into the maternal circulation. Placenta 2003;24:181-190.

8. Alsat E, Wyplosz P, Malassine A, et al. Hypoxia impairs cell fusion and differentiation process in human cytotrophoblast, in vitro. J Cell Physiol 1996;168:346-353.

9. Blumenstein M, Mitchell MD, Groome NP, et al. Hypoxia inhibits activin A production by term villous trophoblast in vitro. Placenta 2002;23:735-741.

10. Kudo Y, Boyd CA, Sargent IL, et al. Hypoxia alters expression and function of syncytin and its receptor during trophoblast cell fusion of human placental BeWo cells: implications for impaired trophoblast syncytialisation in pre-eclampsia. Biochim Biophys Acta 2003;1638: 63-71.

11. Nagamatsu T, Fujii $T$, Kusumi $M$, et al. Cytotrophoblasts upregulate soluble fms-like tyrosine kinase-1 expression under reduced oxygen: an implication for the placental vascular development and the pathophysiology of preeclampsia. Endocrinology 2004;145:4838-4845.

12. Nevo O, Soleymanlou N, Wu Y, et al. Increased expression of sFlt-1 in in vivo and in vitro models of human placental hypoxia is mediated by HIF-1. Am J Physiol Regul Integr Comp Physiol 2006;291:R1085-R1093.

13. Levine RJ, Maynard SE, Qian C, et al. Circulating angiogenic factors and the risk of preeclampsia. N Engl J Med 2004;350:672-683.

14. Maynard SE, Min JY, Merchan J, et al. Excess placental soluble fms-like tyrosine kinase 1 (sFlt1) may contribute to endothelial dysfunction, hypertension, and proteinuria in preeclampsia. J Clin Invest 2003;111:649-658.

15. Hayman R, Warren A, Johnson I, et al. The preliminary characterization of a vasoactive circulating factor(s) in preeclampsia. Am J Obstet Gynecol 2001;184:1196-1203.

16. Anderson NL, Anderson NG. The human plasma proteome: history, character, and diagnostic prospects. Mol Cell Proteomics 2002;1: 845-867.

17. Robinson NJ, Sheikh NA, Baker PN, et al. A serum free in vitro model for the investigation of placental-derived pathogenic factors in preeclampsia. Hypertension Pregnancy 2006;25(Supplement 1): 57 abstract $\mathrm{O075.}$

18. Crocker IP, Tansinda DM, Jones $\mathrm{CJ}$, et al. The influence of oxygen and tumor necrosis factor-alpha on the cellular kinetics of term placental villous explants in culture. J Histochem Cytochem 2004;52: 749-757.

19. Mosmann T. Rapid colorimetric assay for cellular growth and survival: application to proliferation and cytotoxicity assays. J Immunol Methods 1983;65:55-63.

20. Padavala $\mathrm{S}$, Pope $\mathrm{N}$, Baker $\mathrm{P}$, et al. An imbalance between vascular endothelial growth factor and its soluble receptor in placental villous explants of intrauterine growth-restricted pregnancies. J Soc Gynecol Investig 2006;13:40-47.

21. Wareing $M$, Crocker IP, Warren AY, et al. Characterization of small arteries isolated from the human placental chorionic plate. Placenta 2002;23:400-409.

22. Wareing M, Greenwood SL, Taggart MJ, et al. Vasoactive responses of veins isolated from the human placental chorionic plate. Placenta 2003;24:790-796.

23. Green LC, Wagner DA, Glogowski J, et al. Analysis of nitrate, nitrite, and [15N]nitrate in biological fluids. Anal Biochem 1982;126:131-138.

24. Fujikura T, Yoshida J. Blood gas analysis of placental and uterine blood during Cesarean delivery. Obstet Gynecol 1996;87:133-136.

25. Hung TH, Skepper JN, Burton GJ. In vitro ischemia-reperfusion injury in term human placenta as a model for oxidative stress in pathological pregnancies. Am J Pathol 2001;159:1031-1043.

26. Arechavaleta-Velasco F, Hernandez-Guerrero C, Ahued-Ahued R, et al. Evidence of endothelial cytotoxic compounds in placental extracts from preeclamptic women. J Soc Gynecol Investig 2000;7: 114-117.

27. Marchetti $P$, Castedo $M$, Susin SA, et al. Mitochondrial permeability transition is a central coordinating event of apoptosis. J Exp Med 1996;184:1155-1160.

28. Green DR, Reed JC. Mitochondria and apoptosis. Science 1998;281:1309-1312.

29. Desagher S, Osen-Sand A, Nichols A, et al. Bid-induced conformational change of Bax is responsible for mitochondrial cytochrome $c$ release during apoptosis. J Cell Biol 1999;144:891-901.

30. Narita M, Shimizu $S$, Ito $T$, et al. Bax interacts with the permeability transition pore to induce permeability transition and cytochrome $c$ release in isolated mitochondria. Proc Natl Acad Sci USA 1998;95:14681-14686.

31. Basanez G, Nechushtan A, Drozhinin O, et al. Bax, but not Bcl-xL, decreases the lifetime of planar phospholipid bilayer membranes at subnanomolar concentrations. Proc Natl Acad Sci USA 1999;96: 5492-5497.

32. Luo $\mathrm{X}$, Budihardjo $\mathrm{I}$, Zou $\mathrm{H}$, et al. Bid, a $\mathrm{Bcl} 2$ interacting protein, mediates cytochrome $c$ release from mitochondria in response to activation of cell surface death receptors. Cell 1998;94: 481-490.

33. Hu R, Zhou S, Li X. Altered $\mathrm{BCl}-2$ and Bax expression is associated with cultured first trimester human cytotrophoblasts apoptosis induced by hypoxia. Life Sci 2006;79:351-355.

34. Levy R, Smith SD, Chandler K, et al. Apoptosis in human cultured trophoblasts is enhanced by hypoxia and diminished by epidermal growth factor. Am J Physiol Cell Physiol 2000;278:C982-C988.

35. Stepan $\mathrm{H}$, Leo $\mathrm{C}$, Purz $\mathrm{S}$, et al. Placental localization and expression of the cell death factors BNip3 and Nix in preeclampsia, intrauterine growth retardation and HELLP syndrome. Eur J Obstet Gynecol Reprod Biol 2005;122:172-176.

36. Soleymanlou N, Wu Y, Wang JX, et al. A novel Mtd splice isoform is responsible for trophoblast cell death in pre-eclampsia. Cell Death Differ 2005;12:441-452. 
37. Crocker IP, Kenny LC, Thornton WA, et al. Excessive stimulation of poly(ADP-ribosyl)ation contributes to endothelial dysfunction in pre-eclampsia. Br J Pharmacol 2005;144:772-780.

38. Johansen M, Redman CW, Wilkins T, et al. Trophoblast deportation in human pregnancy-its relevance for pre-eclampsia. Placenta 1999;20:531-539.

39. Chua S, Wilkins T, Sargent I, et al. Trophoblast deportation in pre-eclamptic pregnancy. Br J Obstet Gynaecol 1991;98: 973-979.

40. Robinson NJ, Baker $\mathrm{PN}$, Jones $\mathrm{CJ}$, et al. A role for tissue transglutaminase in stabilization of membrane-cytoskeletal particles shed from the human placenta. Biol Reprod 2007;77:648-657.

41. Gupta AK, Rusterholz C, Huppertz B, et al. A comparative study of the effect of three different syncytiotrophoblast microparticles preparations on endothelial cells. Placenta 2005;26: 59-66.

42. Kertesz Z, Hurst G, Ward M, et al. Purification and characterization of a complex from placental syncytiotrophoblast microvillous membranes which inhibits the proliferation of human umbilical vein endothelial cells. Placenta 1999:20:71-79.

43. Kingdom JC, Kaufmann P. Oxygen and placental villous development: origins of fetal hypoxia. Placenta 1997;18:613-621, discussion 623-626.
44. Mayhew TM. Changes in fetal capillaries during preplacental hypoxia: growth, shape remodelling and villous capillarization in placentae from high-altitude pregnancies. Placenta 2003;24:191-198.

45. Odorisio T, Schietroma C, Zaccaria ML, et al. Mice overexpressing placenta growth factor exhibit increased vascularization and vessel permeability. J Cell Sci 2002;115:2559-2567.

46. Suri C, McClain J, Thurston G, et al. Increased vascularization in mice overexpressing angiopoietin-1. Science 1998;282:468-471.

47. Geva E, Ginzinger DG, Zaloudek CJ, et al. Human placental vascular development: vasculogenic and angiogenic (branching and nonbranching) transformation is regulated by vascular endothelial growth factor-A, angiopoietin-1, and angiopoietin-2. J Clin Endocrinol Metab 2002;87:4213-4224.

48. Bujold E, Romero R, Chaiworapongsa $T$, et al. Evidence supporting that the excess of the sVEGFR-1 concentration in maternal plasma in preeclampsia has a uterine origin. J Matern Fetal Neonatal Med 2005;18:9-16.

49. Brownbill P, McKeeman GC, Brockelsby JC, et al. Vasoactive and permeability effects of vascular endothelial growth factor-165 in the term in vitro dually perfused human placental lobule. Endocrinology 2007:148:4734-4744.

50. Martin de Aguilera E, Vila JM, Irurzun A, et al. Endotheliumindependent contractions of human cerebral arteries in response to vasopressin. Stroke 1990;21:1689-1693. 